\title{
Exploiting Single Domain Antibodies as Regulatory Parts to Modulate Monoterpenoid Production in E. coli
}

DOI:

10.1021/acssynbio.0c00375

\section{Document Version}

Final published version

Link to publication record in Manchester Research Explorer

\section{Citation for published version (APA):}

Wilkes, J., Scott-tucker, A., Wright, M., Crabbe, T., \& Scrutton, N. (2020). Exploiting Single Domain Antibodies as Regulatory Parts to Modulate Monoterpenoid Production in E. coli. ACS Synthetic Biology, 9(10), 2828-2839. https://doi.org/10.1021/acssynbio.0c00375

\section{Published in:}

ACS Synthetic Biology

\section{Citing this paper}

Please note that where the full-text provided on Manchester Research Explorer is the Author Accepted Manuscript or Proof version this may differ from the final Published version. If citing, it is advised that you check and use the publisher's definitive version.

\section{General rights}

Copyright and moral rights for the publications made accessible in the Research Explorer are retained by the authors and/or other copyright owners and it is a condition of accessing publications that users recognise and abide by the legal requirements associated with these rights.

\section{Takedown policy}

If you believe that this document breaches copyright please refer to the University of Manchester's Takedown Procedures [http://man.ac.uk/04Y6Bo] or contact uml.scholarlycommunications@manchester.ac.uk providing relevant details, so we can investigate your claim.

\section{OPEN ACCESS}




\title{
Exploiting Single Domain Antibodies as Regulatory Parts to Modulate Monoterpenoid Production in E. coli
}

\author{
Jonathan Wilkes, Anthony Scott-Tucker, Mike Wright, Tom Crabbe, and Nigel S. Scrutton*
}

Cite This: ACS Synth. Biol. 2020, 9, 2828-2839

Read Online

ACCESS

Llll Metrics \& More

Article Recommendations

Supporting Information

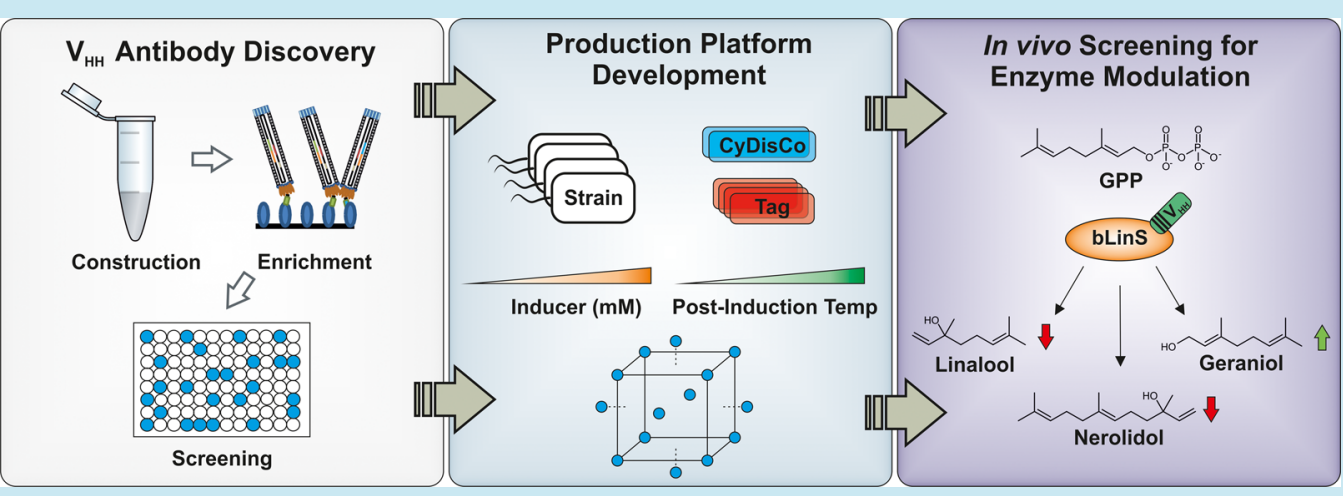

ABSTRACT: Synthetic biology and metabolic engineering offer potentially green and attractive routes to the production of high value compounds. The provision of high-quality parts and pathways is crucial in enabling the biosynthesis of chemicals using synthetic biology. While a number of regulatory parts that provide control at the transcriptional and translational level have been developed, relatively few exist at the protein level. Single domain antibodies (sdAb) such as camelid heavy chain variable fragments $\left(\mathrm{V}_{\mathrm{HH}}\right)$ possess binding characteristics which could be exploited for their development and use as novel parts for regulating metabolic pathways at the protein level in microbial cell factories. Here, a platform for the use of $\mathrm{V}_{\mathrm{HH}}$ as tools in Escherichia coli is developed and subsequently used to modulate linalool production in E. coli. The coproduction of a Design of Experiments (DoE) optimized pBbE8k His ${ }_{6}-\mathrm{V}_{\mathrm{HH}} \mathrm{CyDisCo}$ system alongside a heterologous linalool production pathway facilitated the identification of anti-bLinS $\mathrm{V}_{\mathrm{HH}}$ that functioned as modulators of bLinS. This resulted in altered product profiles and significant variation in the titers of linalool, geraniol, nerolidol, and indole obtained. The ability to alter the production levels of high value terpenoids, such as linalool, in a tunable manner at the protein level could represent a significant step forward for the development of improved microbial cell factories. This study serves as a proof of principle indicating that $\mathrm{V}_{\mathrm{HH}}$ can be used to modulate enzyme activity in engineered pathways within E. coli. Given their almost limitless binding potential, we posit that single domain antibodies could emerge as powerful regulatory parts in synthetic biology applications.

KEYWORDS: single domain antibodies, design of experiments, monoterpenes, enzyme modulators, synthetic biology

$\mathrm{T}_{\mathrm{r}}^{\mathrm{t}}$ he use of single domain antibodies ( $\mathrm{sdAb})$ as novel regulatory parts is an interesting yet unexplored opportunity in synthetic biology workflows for chemicals production. The often fine-specificities and high affinities of antibodies for their binding partners and the availability of display technologies to select for these properties make them attractive molecules for the in-cell targeting of proteins, large molecules, or other structures of interest. Antibodies, and fragments thereof, have been widely exploited as therapeutics, diagnostics, and imaging reagents. When considering the use of antibody fragments within the cell, targeted protein knockdown, ${ }^{1}$ novel transcriptional control, ${ }^{2}$ and targeted protein sequestration and labeling ${ }^{3}$ applications have been developed in mammalian, fish, and insect cells lines. ${ }^{4}$ However, to our knowledge, the use of sdAb to modulate metabolic pathways (e.g., for chemicals production) within microbial chassis has not been reported. In principle, sdAb binding could lead to multiple outcomes: these could include activation or inhibition of pathway enzymes; stabilization of enzymes; interception of small molecule regulation (e.g., allosteric regulation) or reprofiling of catalytic properties (e.g., product outcomes). $\mathrm{sdAb}$ binders possessing one or more of these characteristics may subsequently be exploited as novel regulatory parts that

Received: July 16, 2020

Published: September 14, 2020 
facilitate improved metabolic control at the protein level within microbial cell factories.

One of the most significant barriers to the development of antibody fragments for use in E. coli and other prokaryotic hosts is the inability of these chassis to carry out posttranslational modifications required for function. While the use of sdAb bypasses any requirement for $\mathrm{N}$-glycosylation, the formation of disulfide bonds remains necessary to ensure correct folding of the antibody and retention of function. ${ }^{5}$ Alternative strategies for the development of binding proteins as regulatory components include the use of recombinant binding proteins such as designed ankyrin repeat proteins (DARPins), ${ }^{6}$ monobodies, ${ }^{7}$ affibodies, ${ }^{8}$ anticalins, ${ }^{9}$ and affimers. ${ }^{10}$ The use of such scaffolds is advantageous as they do not require the formation of disulfide bonds, while large libraries of each can be constructed and subsequently enriched in the same manner as antibody libraries. ${ }^{4,11}$ However, sdAb such as camelid heavy chain variable fragments $\left(\mathrm{V}_{\mathrm{HH}}\right)$ possess an elongated binding loop (complementarity determining region 3 ), when compared to those of conventional antibody and recombinant binding protein formats. ${ }^{12}$ This facilitates binding of "cryptic" epitopes on the target antigen, such as those that occur within clefts and cavities, significantly expanding the potential application space of sdAb. The availability of specialized E. coli strains-for example those bearing gor $/ \operatorname{tr} x B$ reductase knockouts, ${ }^{13}$ and plasmid encoded systems such as the CyDisCo catalysts, ${ }^{54-16}$ - facilitates bacterial production of antibody fragments in the cytosol and thus allows their use as regulatory parts.

Microbial cell factories for terpenoid production are attractive targets for in-cell modulation using sdAb. Terpenoids form one of the largest and most diverse classes of natural products, with over 80000 structures reported, and many terpenoids have significant commercial and therapeutic value. The sesquiterpenoid artemisinin has antimalarial properties, ${ }^{17}$ while other terpenoids have applications as flavours, fragrances, antimicrobials, pesticides, and biofuels. ${ }^{18}$ Terpenoids are typically extracted from their natural source or are manufactured by chemical synthesis. ${ }^{19}$ Extraction from natural sources is often low yielding, costly, and dependent on raw material availability. ${ }^{19}$ Chemical synthesis often requires metal catalysts and reaction conditions that can be prohibitive for large scale production. For these reasons, biological production routes using microbial cell factories are potentially attractive, but these routes have yet to be realized for commercial production. To date, a variety of terpenoids have been produced (e.g., artemisinin, farnesene, and limonene) by the construction and development of precursor pathways in tandem with specific terpene synthase/cyclase (TS/C) enzymes. This has been achieved in a variety of hosts including $E$. coli. ${ }^{20-22} \mathrm{New}$ regulatory parts that facilitate increased, altered, or tunable production of terpenoids and their precursors could represent a significant step forward for the development of improved microbial cell factories.

Here we describe a shotgun approach toward the generation and subsequent use of $\mathrm{V}_{\mathrm{HH}}$ as modulators of Streptomyces clavuligerus linalool synthase (bLinS) in E. coli (Figure 1). Following $\mathrm{V}_{\mathrm{HH}}$ discovery, the production of an anti-bLinS $\mathrm{V}_{\mathrm{HH}}$ within the cytosol was improved using a Design of Experiments (DoE) methodology and construction of a partial least-squares regression (PLSR) model. The addition of N-terminal tags alongside the coproduction of the CyDisCo catalysts, $S$. cerevisiae sulfhydryl oxidase Ervlp, and the mature human

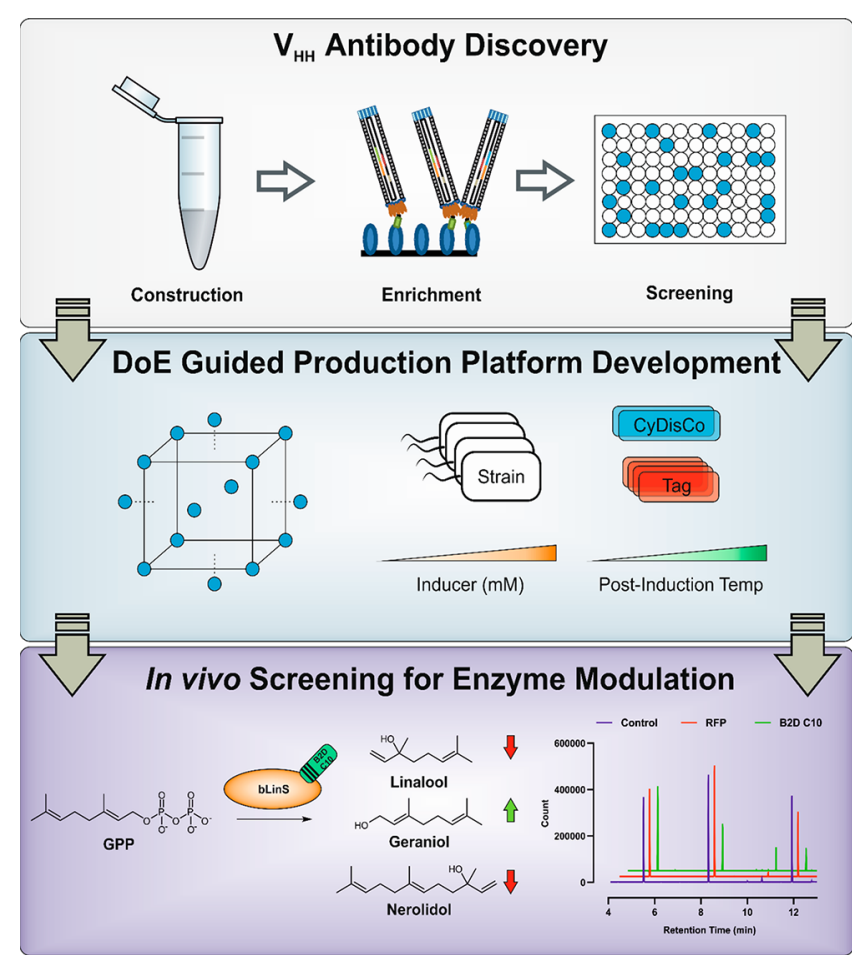

Figure 1. Schematic overview of the workflow followed in this study.

protein disulfide isomerase (PDI) $)^{5,14}$ facilitated significantly improved functional production of a panel of 24 anti-bLinS $\mathrm{V}_{\mathrm{HH}}$. Subsequently, these $\mathrm{V}_{\mathrm{HH}}$ were evaluated for their modulatory capabilities when produced in vivo alongside a heterologous monoterpenoid production pathway. The resultant data revealed the generation of $\mathrm{V}_{\mathrm{HH}}$ bLinS binders that were capable of increasing the linalool titers obtained, as well as the identification of binders capable of inhibiting bLinS, resulting in altered pathway flux. With the ability to routinely generate functional $\mathrm{V}_{\mathrm{HH}}$ against almost any target using in vitro display technologies, this study demonstrates that $\mathrm{sdAb}$ have the potential to be utilized as powerful regulatory parts for a number of synthetic biology applications.

\section{RESULTS AND DISCUSSION}

Construction and Enrichment of anti-bLinS $\mathrm{V}_{\mathrm{HH}}$ Phage Display Libraries. Two immune bLinS $\mathrm{V}_{\mathrm{HH}}$ phage display libraries were constructed following immunization of a llama with purified S. clavuligerus bLinS. A "Bleed 1" library and a "Bleed 2" library, constructed using genetic material isolated following the third and fifth injections were of size 1 and $5 \times 10^{7}$, respectively. Both libraries were enriched using biopanning utilizing two varying wash conditions, in an effort to capture a wider spectrum of bLinS binding affinities. Following the first round of biopanning for each library and wash condition, 95 colonies from each were screened to determine the efficacy of enrichment. Of the 380 total colonies screened, 93\% were identified as bLinS binders by ELISA. Sequencing of these clones yielded 261 unique $\mathrm{V}_{\mathrm{HH}}$ sequences and 158 unique CDR3 sequences within the enriched populations (Supplementary Figure 1).

DoE Optimization of Cytoplasmic $\mathrm{V}_{\mathrm{HH}}$ Production. To utilize the resultant anti-bLinS $\mathrm{V}_{\mathrm{HH}}$ for synthetic biology applications, a platform that facilitated functional production of correctly folded antibodies within the cytoplasm of E. coli was 
required. Given the large number of bLinS binders obtained following library enrichment, a series of optimized conditions for a single $\mathrm{V}_{\mathrm{HH}}$ were first identified. Two of these platforms were subsequently tested on a wider subset of $\mathrm{V}_{\mathrm{HH}}$ in order to determine the efficacy of each. As the most commonly occurring clone within the sequenced population of the enriched libraries, the anti-bLinS $\mathrm{V}_{\mathrm{HH}}$ B2D C10 was chosen for the initial development of the production system.

When considering the optimization of protein production, traditional experimental approaches such as the sequential alteration of individual factors do not always prove time and resource effective. Furthermore, in large multifactor systems key interactions between factors may be missed, resulting in an incomplete understanding of the system in question. This is especially true within biological systems in which complex interactions, such as those between environmental and genetic factors, may have significant effects on a chosen response. Design of Experiments (DoE) is a structured method of experimental design that provides statistical insights to better understand and subsequently optimize complex, multifactor systems. DoE has successfully been used within biological systems in order to explore changes in substrate specificity caused by amino acid substitutions, ${ }^{23}$ to understand and optimize metabolic pathways for the production of various metabolites, ${ }^{24,25}$ and for the evaluation and development of improved translational riboswitches. ${ }^{26}$

Input variables for the experimental design were chosen based on both analysis of the literature and on previously conducted experimental pilot studies (data not included). When evaluating production strains to be used in this work, the inclusion of the $\Delta$ gor $\Delta \operatorname{tr} x B$ double knockout SHuffle T7 Express cells was considered necessary. In an effort to understand the effect of the double knockout system on disulfide bond formation, the regular T7 Express strain, with reducing pathways intact, was also included in the experimental design. Additionally, NiCo21 (DE3) (New England Biolabs) and NEB $10 \beta$ (New England Biolabs) were also included for comparison. The second input variable included in the design was the coproduction of the CyDisCo catalysts yeast sulfhydryl oxidase, Ervlp, and mature human PDI, which were developed for use as an alternative production strategy to $\Delta$ gor $\Delta \operatorname{tr} x B$ double knockout strains. ${ }^{5,14}$ The final categorical input variable explored was the addition of $\mathrm{N}$-terminal tags to aid in protein solubility. ${ }^{27}$ When considering the small size of $\mathrm{V}_{\mathrm{HH}}(\sim 15 \mathrm{kDa})$, and that crystallographic data shows that the $\mathrm{N}$-terminus of the polypeptide chain is adjacent to the CDR loops, ${ }^{28,29}$ the use of large solubility tags have the potential to interfere with the antibody-antigen interaction. Therefore, a series of short, flexible tags; $\mathrm{His}_{6}$ (15 amino acids), $\mathrm{His}_{6}$-TEV (28 amino acids), and $\mathrm{His}_{6} \mathrm{~S}$-tag (46 amino acids), were investigated for their ability to improve production of functional B2D C10 (see Supplementary Table 1 for N-terminal tag amino acid sequences). Finally, inducer concentration and postinduction temperature were included in the design as continuous variables. Both factors are routinely varied ad hoc when looking to optimize protein production in an effort to maximize the rate of protein synthesis while avoiding protein aggregation. ${ }^{30,31}$ The factor levels for each input variable used in the experimental design can be seen in Table 1.

When considering a suitable output response for the experimental design it was decided that measuring the total amount of $\mathrm{V}_{\mathrm{HH}}$ produced would not provide an accurate
Table 1. Input Variables and Associated Factor Levels Chosen for This Experimental Design

\begin{tabular}{|c|c|c|}
\hline input variable & role & factor level \\
\hline strain & categorical & $\begin{array}{l}\text { NEB10 } \beta \text {, NiCo21 (DE3), T7 Express, } \\
\text { SHuffle T7 Express }\end{array}$ \\
\hline CyDisCo & categorical & NO, YES \\
\hline N-terminal Tag & categorical & none, $\mathrm{His}_{6}, \mathrm{His}_{6}$-TEV, $\mathrm{His}_{6} \mathrm{~S}-\mathrm{Tag}$ \\
\hline $\begin{array}{l}\text { inducer } \\
\text { concentration } \\
(\mathrm{mM})\end{array}$ & continuous & $0.1-50$ \\
\hline $\begin{array}{l}\text { postinduction temp } \\
\left({ }^{\circ} \mathrm{C}\right)\end{array}$ & continuous & $16-30$ \\
\hline
\end{tabular}

representation of whether the protein had folded correctly and demonstrated the expected functionality. In an effort to determine the presence of functionally produced $\mathrm{V}_{\mathrm{HH}}$, ELISAs using serially diluted cell lysate and NeutrAvidin captured biotinylated bLinS were performed. The absorbance measured at $630 \mathrm{~nm}$ minus the absorbance at $490 \mathrm{~nm}\left(\Delta A_{630-490}\right)$ was used as a means of comparing the amounts of functionally active B2D C10 present.

PLS Model Building and Interpretation. Following execution of the experimental design, the mean $\Delta A_{630-490}$ values $(n=3)$ were calculated (Supplementary Table 2$)$. The average $\Delta A_{630-490}$ values obtained using lysate at a $1: 1000$ dilution were then used to construct a partial least-squares (PLS) regression model using each of the effects included in the custom design. Model validation was performed using leave-one-out cross validation (LOOCV) and the statistically inspired modification of the PLS method (SIMPLS) algorithm. ${ }^{32}$ The optimal number of factors (latent variables) within each model was determined by choosing the fit which minimized the RM-PRESS statistic. For the first iteration of the PLS model, which included all main effects, all interactions, and powered terms for continuous variables, it was determined that two factors were optimal $(\mathrm{RM}-\mathrm{PRESS}=0.866)$. While a cumulative $R^{2} Y$ value of 0.899 indicated that the model explained a significant amount of variation in the $Y$ variables, the predictive ability of the model, as determined by the cumulative $Q^{2}$ value of 0.437 , suggested that the model could be further improved. Of the 67 terms included in the initial model 34 were identified as influential, as determined by the term having a variable importance in projection (VIP) statistic greater than 0.8 .

To improve the predictive ability of the PLS model each of the terms with a VIP statistic greater than 0.8 (Figure 2A), including any nonsignificant main effect terms contained within significant interaction terms, were taken forward to construct a second PLS model. The resultant model, which utilized three factors (RM-PRESS $=0.789)$, demonstrated improved predictive power as exemplified by a cumulative $R^{2} Y$ value of 0.929 and a cumulative $Q^{2}$ value of 0.702 . A series of diagnostic plots were generated to further demonstrate the model was of sufficient quality for predictive use (Supplementary Figure 2). The actual by predicted plot (Supplementary Figure 2A) confirmed that the data were largely well predicted by the model. While the residual by predicted plot (Supplementary Figure 2B), residual by row (Supplementary Figure 2C), and residual normal quantile (Supplementary Figure 2D) showed a balanced distribution, with the exception of the edge effect seen in the residual by predicted plot (Supplementary Figure 2B) for experiments which had $\Delta A_{630-490}$ values of 0 , that is, no bound $\mathrm{V}_{\mathrm{HH}}$ was detected. 
A

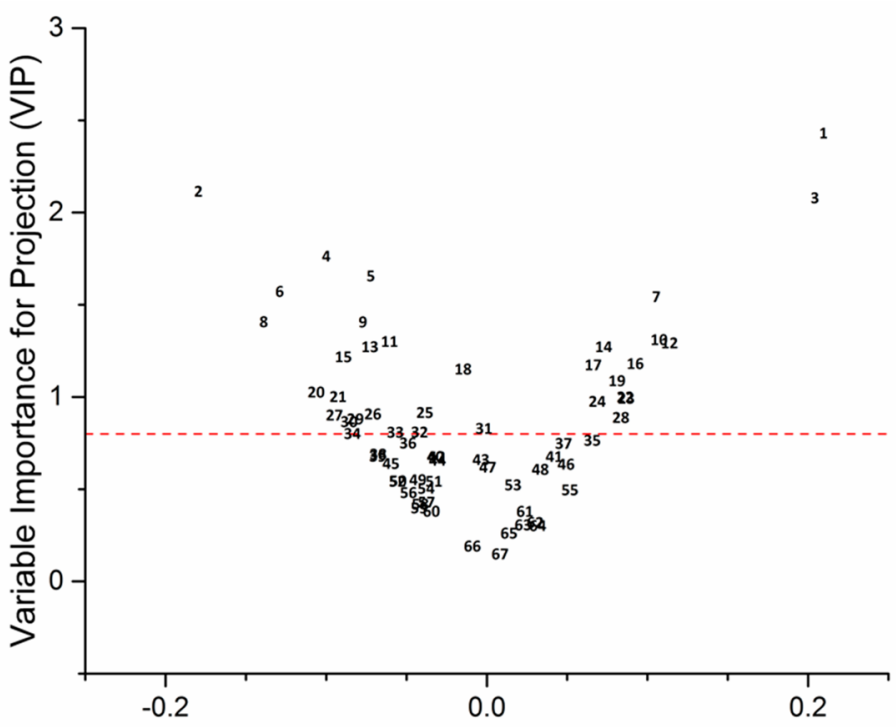

Model Coefficients for Centered and Scaled Data

\begin{tabular}{|c|c|c|c|c|c|}
\hline 1 & $\mathrm{~N}$-term tag[His]*CyDisCo[Yes] & 12 & CyDisCo[Yes] ${ }^{*}$ Strain[NEB10 $\left.\beta\right]$ & 24 & $\mathrm{~N}$-term tag[His]*Strain[NiCo21(DE3)] \\
\hline 2 & Inducer Conc (mM)*Strain[SHuffle T7] & 13 & CyDisCo[No] & 25 & CyDisCo[Yes] ${ }^{*}$ Strain[T7 Express] \\
\hline 3 & N-term tag[His + S-Tag] ${ }^{*}$ Strain[SHuffle T7] & 14 & CyDisCo[Yes] & 26 & Inducer Conc (mM)*Strain[NiCo21(DE3)] \\
\hline 4 & CyDisCo[Yes]*Inducer Conc (mM) & 15 & $\mathrm{~N}$-term tag[His + S-Tag $]^{*}$ Inducer Conc (mM) & 27 & $\mathrm{~N}$-term tag[His TEV]*Strain[NiCo21(DE3)] \\
\hline 5 & Inducer Conc $(\mathrm{mM})(0.1,50)$ & 16 & Strain[SHuffle T7] & 28 & Strain[NiCo21(DE3)] ${ }^{*}$ Post Induction Temp $\left({ }^{\circ} \mathrm{C}\right)$ \\
\hline 6 & $\mathrm{~N}$-term tag[His]*Inducer Conc (mM) & 17 & CyDisCo[Yes]*Strain[SHuffle T7] & 29 & $\mathrm{~N}$-term tag[None]*CyDisCo[Yes] \\
\hline 7 & $\mathrm{~N}$-term tag[His] & 18 & $\mathrm{~N}$-term tag $[\mathrm{None}]^{*} \mathrm{CyDis} \mathrm{Co}[\mathrm{No}]$ & 30 & Strain $[$ NEB1OB $]{ }^{*}$ Post Induction Temp $\left({ }^{\circ} \mathrm{C}\right)$ \\
\hline 8 & Post Induction Temp $\left({ }^{\circ} \mathrm{C}\right){ }^{*}$ Post Induction Temp $\left({ }^{\circ} \mathrm{C}\right)$ & 19 & N-term tag[His]*Strain[SHuffle T7] & 31 & $\mathrm{~N}$-term tag[None]*Strain[T7 Express] \\
\hline 9 & $\mathrm{~N}$-term tag[None] & 20 & $\mathrm{~N}$-term tag[None]*Strain[SHuffle T7] & 32 & CyDisCo[No]"Strain[T7 Express] \\
\hline 10 & $\mathrm{~N}$-term tag[His]*Strain[NEB10ß] & 21 & CyDisCo[No] ${ }^{*} \operatorname{Strain}[\mathrm{NEB} 10 \beta]$ & 33 & $\mathrm{~N}$-term tag[His TEV] ${ }^{*} \mathrm{CyDisCo}[\mathrm{No}]$ \\
\hline 11 & Strain[T7 Express] & 22 & N-term tag[ $[\text { one }]^{*}$ Inducer Conc $(\mathrm{mM})$ & 34 & $\mathrm{~N}$-term tag $[\mathrm{His}]^{*} \mathrm{CyDisCo}[\mathrm{No}]$ \\
\hline 12 & CYDisCo[Yes]*Strain[NEB10B] & 23 & Inducer Conc $(\mathrm{mM}) * \operatorname{Strain}[\mathrm{NEB} 10 \mathrm{~B}]$ & & \\
\hline
\end{tabular}

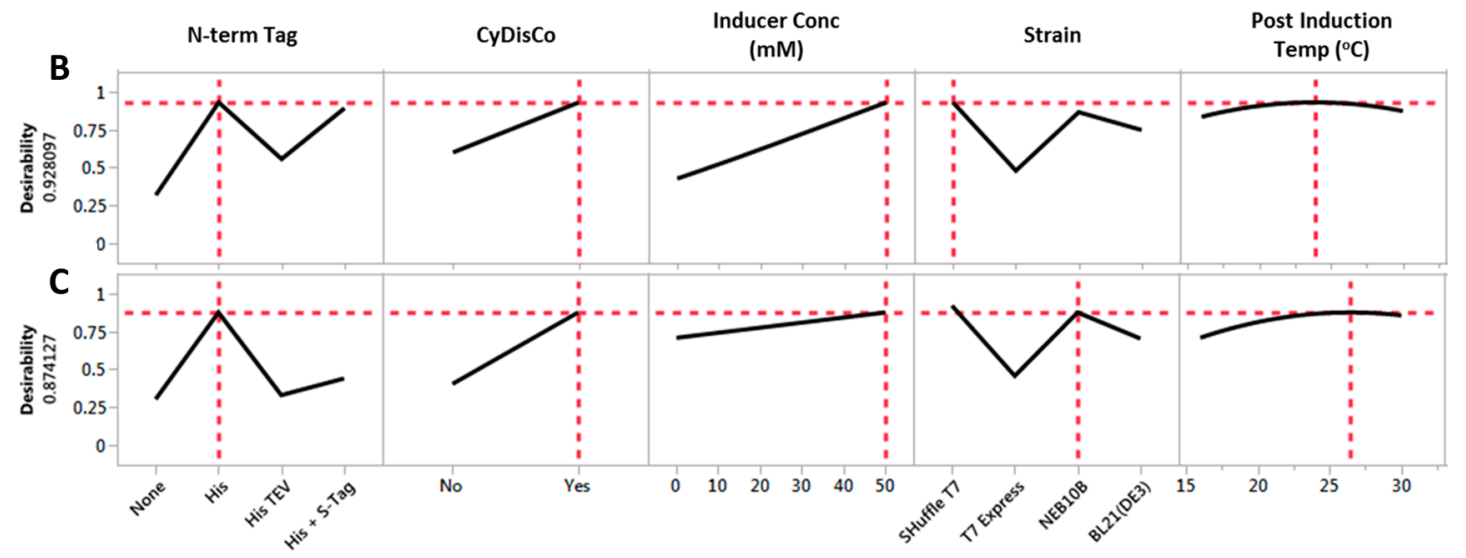

Figure 2. Variable importance in projection (VIP) against model coefficients for centered and scaled data plot. Standardized coefficients indicate if the term has a positive or negative impact in the model. Terms with a VIP score greater than 0.8 (red dashed line) were deemed as influential in the explanatory model for $\mathrm{V}_{\mathrm{HH}}$ binding as determined using the average $\Delta A_{630-490}(1: 1000)$. Interaction terms are denoted by an asterisk (*). (B and C) Prediction profiler plots for functional production of the anti-bLinS $V_{\mathrm{HH}}$ B2D C10 under optimized conditions.

To identify a combination of the input factors that yielded optimal functional B2D C10 production, the predictive power of the PLS model was used. The resultant prediction profiler plots showed the combination of factors that yielded the most desirable $\Delta A_{630-490}(1: 1000)$ output, which were assigned a desirability score between 0 and 1 (Figure 2B,C). The most desirable factor combination, that is, the combination with the highest predicted $\Delta A_{630-490}(1: 1,000)$ value had a score of 0.928. This combination utilized an $\mathrm{N}$-terminal $\mathrm{His}_{6}$ tag, the coproduction of the CyDisCo catalysts and the use of SHuffle T7 Express cells, with induction using $50 \mathrm{mM}$ arabinose and a reduction of the incubation temperature to $23.7^{\circ} \mathrm{C}$ following induction (Figure 2B). When considering optimal production levels in alternative strains, the addition of an $\mathrm{N}$-terminal $\mathrm{His}_{6}$ tag, and the coproduction of the CyDisCo catalysts in NEB $10 \beta$ following induction with $50 \mathrm{mM}$ arabinose and incubation at $26.6{ }^{\circ} \mathrm{C}$, had the next highest desirability score of 0.874 (Figure 2C).

Evaluating the Production of anti-bLinS $\mathrm{V}_{\mathrm{HH}}$ Using Optimized Conditions. Following the successful identification of two conditions that could be used to improve the cytoplasmic production of the anti bLinS $\mathrm{V}_{\mathrm{HH}}$ B2D C10, both were tested for their ability to produce other anti-bLinS $\mathrm{V}_{\mathrm{HH} .} \mathrm{A}$ selection of 24 anti-bLinS $\mathrm{V}_{\mathrm{HH}}$ possessing the most commonly 

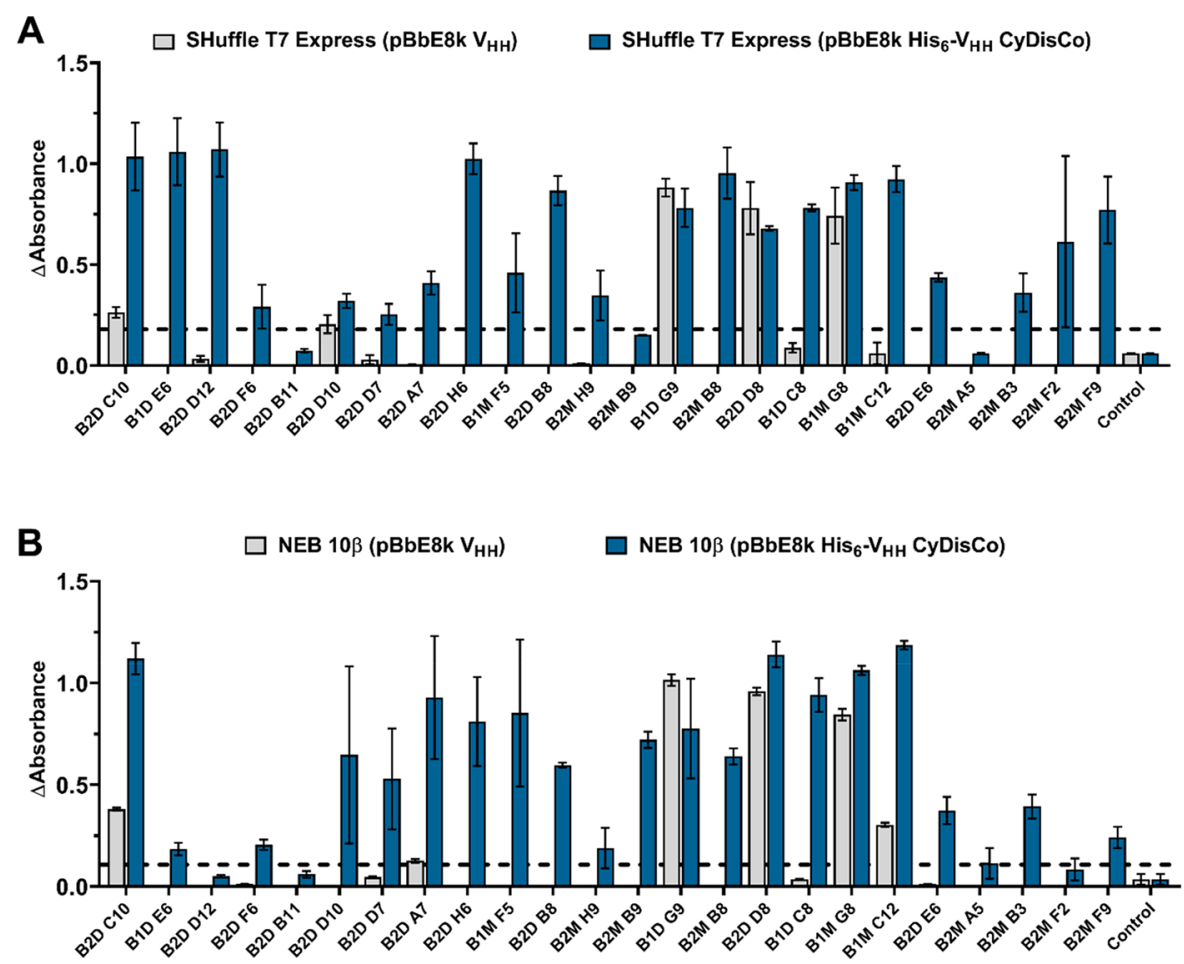

Figure 3. Comparison of functionally produced anti-bLinS $\mathrm{His}_{6}-\mathrm{V}_{\mathrm{HH}}$ under optimized production conditions in SHuffle T7 Express (A) and NEB $10 \beta(\mathrm{B})$, as determined by ELISA using clarified cell lysate. The threshold for functional production of each $\mathrm{V}_{\mathrm{HH}}$ was determined as a 3-fold increase of the signal observed for the pBbE8k His-RFP lysate control (indicated by the dotted line). $\Delta$ Absorbance was determined as the absorbance measured at $630 \mathrm{~nm}$ minus the absorbance at $490 \mathrm{~nm}\left(\Delta A_{630-490}\right)$. All measurements were performed in biological triplicate $(n=3)$ with the standard deviation reported.

occurring CDR3 sequences were chosen for further evaluation of the cytoplasmic production platform (see Supplementary Table 3 for aligned sequences). These $\mathrm{V}_{\mathrm{HH}}$ were cloned into the $\mathrm{pBbE} 8 \mathrm{k} \mathrm{His}_{6}$-RFP CyDisCo vector and produced using the optimized conditions described above. The cell lysate was then used to perform an ELISA against immobilized biotinylated bLinS in order to identify functional production of the $\mathrm{V}_{\mathrm{HH}}$ in question. When using the optimized conditions functional production of 21 out of $24(87.5 \%)$ anti-bLinS $\mathrm{V}_{\mathrm{HH}}$, as determined by a $\Delta \mathrm{A}_{630}-\mathrm{A}_{490}$ value 3 -fold greater than that of the pBbE8k His-RFP lysate control, was observed (Figure 3). In comparison, when the same anti-bLinS $\mathrm{V}_{\mathrm{HH}}$ were produced in the absence of an $\mathrm{N}$-terminal $\mathrm{His}_{6}$ tag and $\mathrm{CyDisCo}$ catalysts only 5 of the $24 \mathrm{~V}_{\mathrm{HH}}$ selected (20.1\%) were functionally produced (Supplementary Table 4). These results suggest that the optimized conditions identified herein represent a suitable platform upon which $\mathrm{V}_{\mathrm{HH}}$ may be used as binders within the cytosol of E. coli.

Strain Comparison of In Vivo Linalool Production. During the optimization of anti-bLinS $\mathrm{V}_{\mathrm{HH}}$ production in $E$. coli using a DoE methodology, the most suitable strains were found to be SHuffle T7 Express and NEB 10 $\beta$. Given that the choice of strain, carbon source, and redox balance within the cell have been shown to affect limonene production, ${ }^{33}$ the ability of SHuffle T7 Express and NEB $10 \beta$ cells to produce linalool using the pJBEI-6410 bLinS plasmid (Figure 4A) was first investigated in order to establish which strain was better suited for linalool production in the absence of anti-bLinS $\mathrm{V}_{\mathrm{HH}}$. The strains NEB5 $\alpha$, BL21 (DE3), and T7 Express were also included for comparison.

Of the strains evaluated, NEB $10 \beta$ produced the greatest quantity of linalool (Figure 4B). The use of $1 \%(\mathrm{v} / \mathrm{v})$ glycerol as the primary carbon source facilitated the production of 87.1 $\pm 6.1 \mathrm{mg} \mathrm{L}_{\mathrm{org}}^{-1}$ of linalool, while $1 \%(\mathrm{v} / \mathrm{v})$ glucose resulted in $82.8 \pm 35.4 \mathrm{mg} \mathrm{L}_{\mathrm{org}}{ }^{-1}$. SHuffle T7 Express produced the least linalool of the strains evaluated, achieving linalool titers of 0.2 $\pm 0.1 \mathrm{mg} \mathrm{L}_{\text {org }}^{-1}$ and $0.2 \pm 0.3 \mathrm{mg} \mathrm{L}_{\text {org }}^{-1}$ when utilizing glucose and glycerol $(1 \% \mathrm{v} / \mathrm{v})$ as the carbon source, respectively. The relatively poor linalool titers produced by SHuffle T7 Express may be due to the altered redox pathways present in this strain. $^{13}$

As NEB $10 \beta$ was a suitable production host for anti-bLinS $\mathrm{V}_{\mathrm{HH}}$ and also achieved the highest linalool titers under the conditions tested, this strain was the obvious choice to carry forward for future experiments, with glycerol as the primary carbon source. To evaluate the modulatory effects of antibLinS $\mathrm{V}_{\mathrm{HH}}$ on the production of linalool, conditions that were developed to favor the production of anti-bLinS $\mathrm{V}_{\mathrm{HH}}$ ( $\mathrm{His}_{6}$ tag, CyDisCo catalysts, NEB 10 $\beta, 50 \mathrm{mM}$ arabinose, and $26.6^{\circ} \mathrm{C}$ postinduction temperature) were taken forward for in vivo experiments.

In Vivo Modulation of bLinS Using anti-bLinS $\mathrm{V}_{\mathrm{HH}}$ During initial efforts to produce anti-bLinS $\mathrm{V}_{\mathrm{HH}}$ alongside the heterologous linalool production pathway, upon induction of cells transformed with both pJBEI-6410 bLinS and pBbE8k $\mathrm{His}_{6}-\mathrm{V}_{\mathrm{HH}} \mathrm{CyDisCo}$, cell growth was severely impaired, preventing reliable analysis of the terpenoid products. This might reflect an increased metabolic burden as a result of $\mathrm{V}_{\mathrm{HH}}$ overproduction. Of the factors investigated during the DoE guided optimization of $\mathrm{B} 2 \mathrm{D} \mathrm{C} 10$ production in NEB $10 \beta$, it was noted that inducer concentration had a less significant impact on the Prediction Profiler desirability score than altering the choice of $\mathrm{N}$-terminal tag or removing the $\mathrm{CyDisCo}$ catalysts. In an effort to reduce metabolic burden, the decision 
A
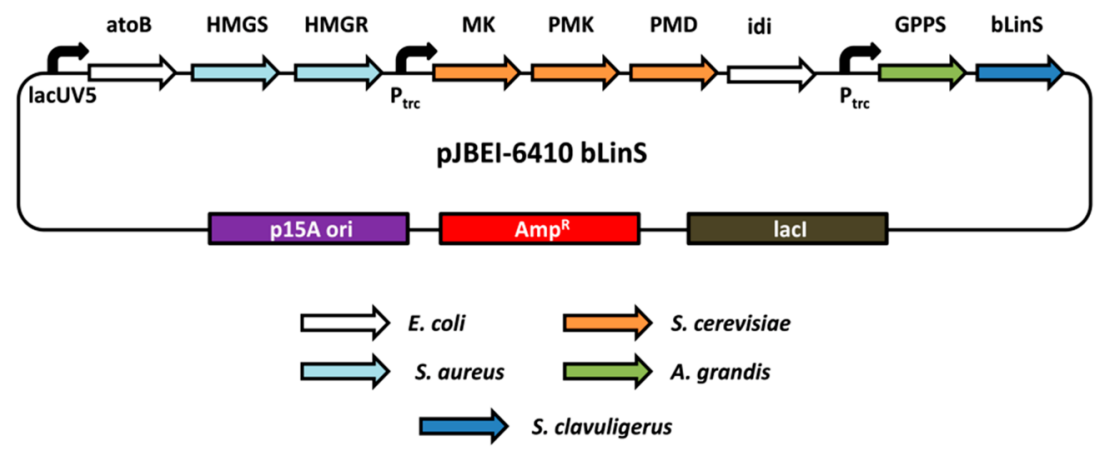

B

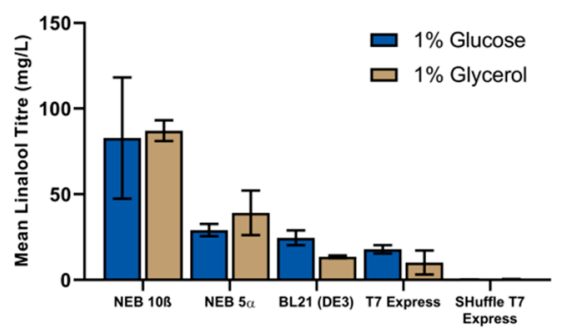

D

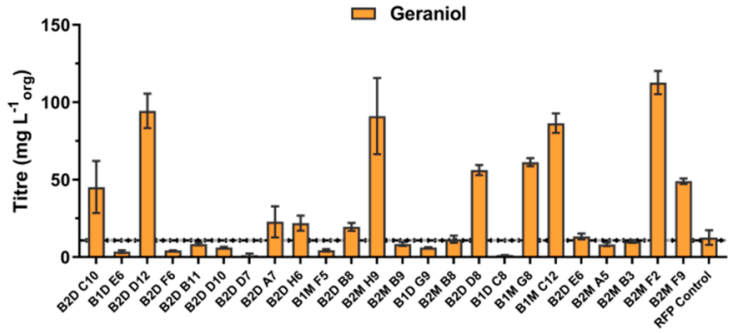

C

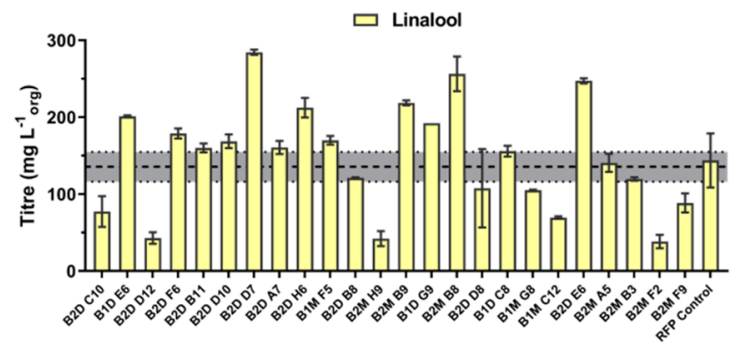

E

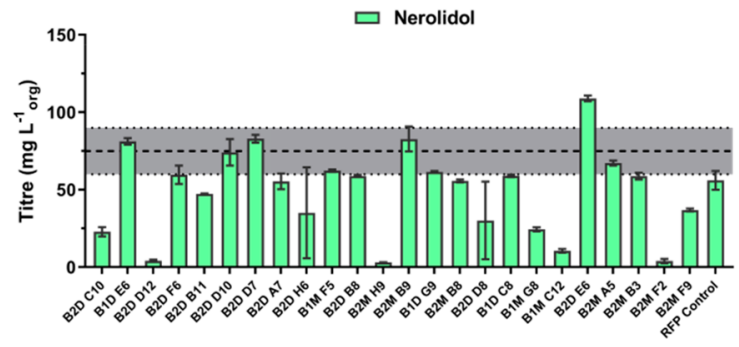

Figure 4. In vivo modulation of a heterologous linalool production pathway in E. coli (A) Diagrammatic representation of the pJBEI-6410 bLinS plasmid containing the heterologous mevalonate (MVA) pathway, Abies grandis geranyl pyrophosphate synthase (GPPS), and Streptomyces clavuligerus linalool synthase (bLinS) required for the production of linalool in E. coli. The genes contained within the heterologous MVA pathway are as follows: acetyl-CoA acetyltransferase (atoB), hydroxymethylglutaryl-CoA synthase (HMGS), hydroxymethylglutaryl-CoA reductase (HMGR), mevalonate kinase (MK), phosphomevalonate kinase (PMK), phosphomevalonate decarboxylase (PMD) and isopentenyl diphosphate isomerase (idi). (B) Strain comparison for the production of linalool using either $1 \%(\mathrm{v} / \mathrm{v})$ glycerol or glucose as the primary carbon source in phosphate buffered TB media. Monoterpenoid production was induced by the addition of $100 \mu \mathrm{M}$ IPTG at an OD $600 \sim 0.6$. Cultures were incubated at $30{ }^{\circ} \mathrm{C}$ for $72 \mathrm{~h}$ following induction. All measurements were obtained in biological triplicate $(n=3)$. (C,D,E) Titers of terpenoid products obtained from the organic overlay of NEB 10B transformed with both the pJBEI-6410 bLinS and pBbE8k His- $\mathrm{V}_{\mathrm{HH}}$ CyDisCo plasmids. The dashed line represents the average titer observed for the pJBEI-6410 bLinS control, with the standard deviation highlighted in gray. An additional pJBEI-6410 bLinS and pBbE8k His-RFP CyDisCo control (RFP Control) was also included. All measurements were performed in biological triplicate $(n=3)$.

was made to reduce the amount of arabinose added for the induction of $\mathrm{V}_{\mathrm{HH}}$ production.

When the concentration of arabinose added was reduced to $2 \mathrm{mM}$, reliable growth of pJBEI-6410 bLinS and pBbE8k His ${ }_{6}-$ $\mathrm{V}_{\mathrm{HH}}$ CyDisCo transformed NEB $10 \beta$ was observed. Furthermore, altered linalool titers were observed in a number of cases when comparing the pJBEI-6410 bLinS only (135.5 \pm $19.4 \mathrm{mg} \mathrm{L}^{-1}$ ) and pJBEI-6410 bLinS + pBbE8k His 6 -RFP CyDisCo $\left(143.8 \pm 35.1 \mathrm{mg} \mathrm{L}^{-1}\right)$ controls. The coproduction of $\mathrm{V}_{\mathrm{HH}}$ B2D C10, B2D D12, B2M H9, B1M C12, and B2M F2 resulted in significantly reduced linalool titers, suggesting that these $\mathrm{V}_{\mathrm{HH}}$ function as inhibitors of bLinS in the cytoplasm. Coproduction of anti-bLinS $\mathrm{V}_{\mathrm{HH}}$ B1D E6, B2D D7, B2D H6, B2M B9, B1D G9, B2M B8, and B2D E6 facilitated increased linalool titers; with B2D D7 resulting in an approximate 2-fold increase in linalool production $\left(284.5 \pm 3.3 \mathrm{mg} \mathrm{L}^{-1}\right)$. This suggests that these $\mathrm{V}_{\mathrm{HH}}$ stabilize the enzyme/enzymesubstrate complex within the cytoplasm.

In addition to the production of linalool, geraniol and nerolidol were also detected by GC-MS (Figure 4D,E). The production of geraniol by $E$. coli containing a heterologous MVA pathway and GPPS in the absence of a geraniol synthase gene was reported previously and identified to be a result of promiscuous activity of native enzymes including alkaline phosphatase (PhoA). ${ }^{21,34}$ In this investigation, increased geraniol titers were observed for each of the previously identified inhibitory $\mathrm{V}_{\mathrm{HH}}$ (Figure 4D). The increased geraniol production in vivo is likely a result of the changing flux within the biosynthetic pathway occurring in response to the $\mathrm{V}_{\mathrm{HH}^{-}}$ bLinS binding. The inhibitory effect of the anti-bLinS $\mathrm{V}_{\mathrm{HH}}$ is likely to create a bottleneck within the pathway, resulting in the accumulation of GPP precursors IPP and DMAPP within the 


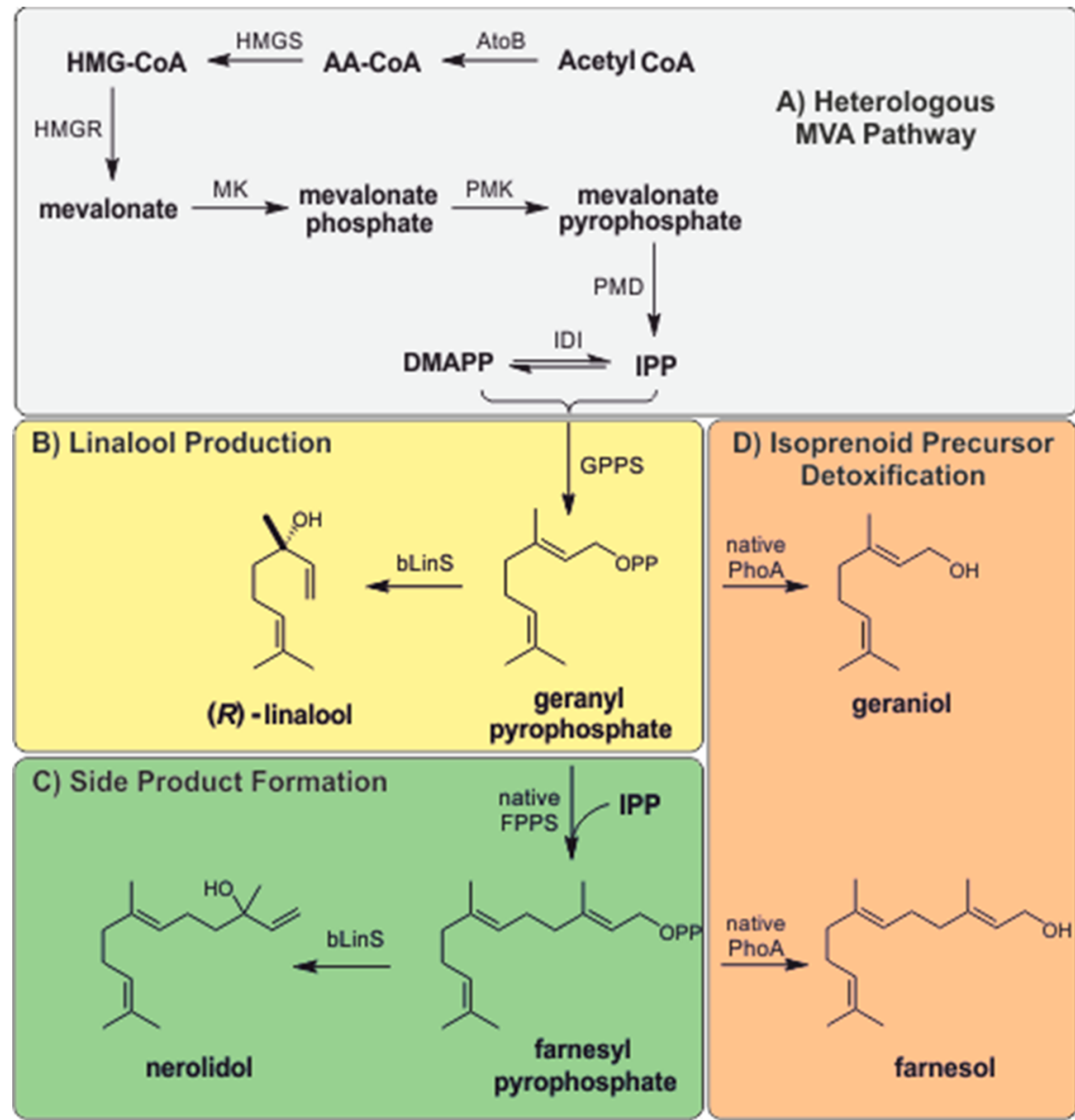

Figure 5. Proposed terpenoid production pathways occurring within the cytoplasm of $E$. coli transformed with pJBEI-6410 bLinS in the absence of inhibitory $\mathrm{V}_{\mathrm{HH}}(\mathrm{A}, \mathrm{B}, \mathrm{C})$ and in the presence of inhibitory $\mathrm{V}_{\mathrm{HH}}$ (D). Upon production of inhibitory $\mathrm{V}_{\mathrm{HH}}$ within the cytoplasm, it is proposed a number of native enzymes, including alkaline phosphatase (PhoA), are recruited to prevent accumulation of the toxic isoprenoid precursors IPP and DMAPP.

cell, both of which have been shown to be cytotoxic in $E$. coli. ${ }^{35-37}$ As a result of increased GPP availability, conversion to geraniol by PhoA and other unidentified endogenous pathways ${ }^{21}$ would alleviate toxic effects of isoprenoid precursor accumulation (Figure 5).

$S$. clavuligerus bLinS has also been shown to have nerolidol synthase activity in the presence of the $\mathrm{C}_{15}$ sesquiterpenoid precursor farnesyl pyrophosphate (FPP). ${ }^{38}$ Strains of E. coli are capable of producing FPP using native enzymes. ${ }^{39}$ Nerolidol titers were reduced in the presence of each of the anti-bLinS $\mathrm{V}_{\mathrm{HH}}$, with the notable exception of B2D E6 which instead conferred increased titers of nerolidol detected (Figure 4E). This could suggest that the majority of the $\mathrm{V}_{\mathrm{HH}}$ selected interact with bLinS near the active site, and thereby impair access of the longer $\mathrm{C}_{15}$ substrate. With the $\mathrm{V}_{\mathrm{HH}}$ B2D E6, however, the ability of bLinS to turn over both the $\mathrm{C}_{10}$ and $\mathrm{C}_{15}$ substrates was improved. The reduction of nerolidol titers was most significant in the presence of the $\mathrm{V}_{\mathrm{HH}}$ B2D C10, B2D D12, B2M H9, B1M C12, and B2M F2, when compared to the pJBEI-6410 bLinS only and pJBEI-6410 bLinS + pBbE8k RFP CyDisCo controls, further suggesting that these $\mathrm{V}_{\mathrm{HH}}$ are indeed inhibitors of bLinS.

Finally, it was also observed that the titers of indole, an intracellular signaling compound that was identified previously as a potential inhibitor of isoprenoid pathway activity, ${ }^{40}$ were significantly reduced following the production of each anti-
bLinS $\mathrm{V}_{\mathrm{HH}}$ as well as for the pBbE8k His ${ }_{6}$-RFP CyDisCo control (Supplementary Figure 3). This suggests that the presence of the second plasmid and the coproduction of the CyDisCo catalysts may alleviate potential toxicity issues attributed to the accumulation of toxic isoprenoid precursors and monoterpenoids within the cell, and thus lower the observed indole titers. We propose that this could be due to a more gradual accumulation of isoprenoid precursors and monoterpenoids within the chassis, due to increased metabolic burden within cells bearing both plasmids, resulting in a muted isoprenoid stress response.

In this proof of principle study, we have demonstrated that $\mathrm{V}_{\mathrm{HH}} \mathrm{sdAb}$ can be used as novel regulatory parts to modulate engineered metabolic pathways in $E$. coli. We have also demonstrated that a DoE workflow can be used to optimize expression of $\mathrm{V}_{\mathrm{HH}}$ binders in $E$. coli, which can be readily adapted for use with other microbial species. Using this workflow, and with continued development, the generation of $\mathrm{sdAb}$ with a variety of regulatory functionalities is feasible. This could open up new opportunities to regulate engineered pathways through controlled expression of highly specific $\mathrm{V}_{\mathrm{HH}}$ binders. Here, we have described inhibitors and activators of linalool production but other applications are also envisaged. For example, high affinity binders with no observed modulatory effect on bLinS could be used as a scaffold or tag to facilitate colocalization of enzymes. In monoterpene 
producing pathways, GPPS and monoterpene synthases are known to be rate limiting enzymes, ${ }^{41,42}$ and their colocalization through tethering has been shown to increase the monoterpenoid titers obtained. ${ }^{43}$ When considering applications of inhibitory binders, targeted knockdown of a variety of proteins have been explored in eukaryotic systems, ${ }^{1,4,44}$ demonstrating that $\mathrm{sdAb}$ are viable knockdown alternatives to gene editing technologies and RNAi based approaches. This work shows that targeted knockdown using sdAb is also possible in E. coli, facilitating the development of novel repression or feedback control systems with synthetic biology applications. Furthermore, the ability to alter pathway flux, as described here for the production of geraniol via native detoxification pathways following bLinS inhibition, is also significant. An improved ability to redirect metabolic pathways in an inducible, tunable manner provides a platform for the development of a new generation of microbial cell factories. As such, the $V_{H H}$ platforms and associated workflows described in this communication could now facilitate broad uptake of $\mathrm{V}_{\mathrm{HH}}$ antibodies as novel parts for metabolic pathways engineering and regulation.

\section{METHODS}

Vector Construction. All primers/oligonucleotide sequences used in this study can be found in Supplementary Table 5. Purification of PCR products and agarose gel extractions were performed using a NucleoSpin Gel and PCR Clean-up Kit (Macherey-Nagel). All constructs were sequenced by Eurofins Genomics to ensure correct sequence identity.

For the production of bLinS used in in vitro assays the codon optimized Streptomyces clavuligerus linalool synthase (bLinS) gene was cloned between the $\mathrm{NcoI}$ and $\mathrm{XhoI}$ restriction sites of the pETM11 vector as described previously. ${ }^{38}$ The in vivo production of biotinylated bLinS was achieved using Lucigen's Expresso Biotin Cloning and Expression System. S. clavuligerus bLinS from pETM11 bLinS was PCR amplified using Expresso bLinS Biotinylation Fw \& Expresso bLinS Biotinylation Rv primers. Biotin XCell $\mathrm{F}^{\prime}$ chemically competent cells (Lucigen) were transformed with the purified PCR product and the linear pAviTag C-His vector. Colonies were screened by cPCR using pRham Fw and pETite Rv to determine the presence of the desired insert.

For the production of linalool in vivo, bLinS was cloned into the pJBEI-6410 vector. $^{45}$ pJBEI-6410, a gift from Taek Soon Lee (Addgene plasmid \#47049; http://n2t.net/ addgene:47049), was PCR linearized using primers pJBEI6410_Syn_open_3' and pJBEI-6410_Syn_open_5'. Codon optimized bLinS was PCR amplified from pETM11 bLinS, using primers LinS+6410 Fw and LinS+6410 Rv. The purified PCR products were then cloned using an InFusion Cloning Kit (Clontech). Following the transformation of Stellar Competent cells (Clontech), cPCR using Syn_Seq_Fw and Syn_Seq_Rv primers was performed to confirm the presence of the inset. The resultant plasmid was subsequently referred to as pJBEI-6410 bLinS.

For the construction of vectors containing the CyDisCo catalyst system, ${ }^{5,14-16}$ codon optimized genes for the $S$. cerevisiae sulfhydryl oxidase Ervlp and mature human PDI were synthesized (Integrated DNA Technologies). The CyDisCo catalyst cassette included bespoke 5' UTR and RBS sequences designed using the Ribosome Binding Calculator $^{46,47}$ and the rhaB promoter ${ }^{48}$ (coding sequences can be found in Supplementary Table 6). Initially, the synthesized CyDisCo fragment was cloned into the PCR linearized pBbE8k vector ${ }^{49}$ (pBbE8k CyDisCo Fw \& $\mathrm{Rv}$ primers, Supplementary Table 5) using an In-Fusion Cloning kit (Clontech). The resultant plasmid was subsequently referred to as pBbE8k RFP Rha-CyDisCo. For use in the DoE vectors the CyDisCo system was instead cloned into the $\mathrm{pBbE8k}$ vector under the control of the araBAD promoter, such that the $\mathrm{RFP} / \mathrm{V}_{\mathrm{HH}}$, Ervlp, and PDI proteins were produced in a tricistronic manner. This was achieved by PCR amplification of pBbE8k RFP Rha-CyDisCo using pBbE8k TriCistronic CyDisCo $\mathrm{Fw}$ and $\mathrm{Rv}$ primers. The purified PCR product was then phosphorylated and ligated using KLD Enzyme Mix (New England Biolabs), prior to transformation of NEB $10 \beta$ competent cells. The resultant plasmid was referred to as $\mathrm{pBbE8k}$ RFP CyDisCo.

For the addition of $\mathrm{N}$-terminal tags to the pBbE8k RFP CyDisCo expression vector, the plasmid was digested using the NdeI endonuclease in tandem with Antarctic phosphatase (New England Biolabs). N-terminal $\mathrm{His}_{6}, \mathrm{His}_{6}-\mathrm{TEV}$ and $\mathrm{His}_{6}$ S-tags with $15 \mathrm{bp}$ overhangs were designed and synthesized (Integrated DNA Technologies) such that there was complementarity to the digested backbone and that the NdeI restriction site was restored at the $3^{\prime}$ end of the inset following cloning. In-Fusion Cloning (Clontech) was used to assemble the tagged RFP tricistronic CyDisCo expression cassette.

Select $\mathrm{V}_{\mathrm{HH}}$ coding sequences were PCR amplified using $\mathrm{V}_{\mathrm{HH}}$ NdeI Fw and $\mathrm{V}_{\mathrm{HH}}$ XhoI Rv primers. The purified $\mathrm{V}_{\mathrm{HH}}$ insert and $\mathrm{pBbE8k}$ tagged-RFP CyDisCo vector variants were digested using NdeI and XhoI endonucleases and subsequently gel purified using the NucleoSpin kit, prior to ligation using T4 DNA ligase (New England BioLabs). NEB $10 \beta$ cells were transformed with the ligation mixture and plated on $\mathrm{LB}$ kanamycin $\left(50 \mu \mathrm{g} \mathrm{mL}^{-1}\right)$ plates supplemented with $1 \mathrm{mM}$ arabinose, such that instances of vector background could be more easily identified by the presence of red fluorescent protein producing colonies. cPCR using pBbE8k Seq Fw and $\mathrm{Rv}$ primers was performed to identify colonies containing the correct size of insert, which were subsequently sent for Sanger sequencing (Eurofins Genomics).

Protein Production. bLinS was produced and purified as described previously. ${ }^{38}$ Biotinylated bLinS was produced in vivo using Lucigen's Expresso Biotin Cloning and Expression System. Following the construction of the pAviTag C-His bLinS vector, a single colony was used to inoculate small scale cultures of LB broth (ForMedium) supplemented with kanamycin $\left(30 \mu \mathrm{g} \mathrm{mL}^{-1}\right)$, which were incubated at $37{ }^{\circ} \mathrm{C}$ overnight. These were used to inoculate $(1: 100) 6 \times 1 \mathrm{~L}$ cultures which were grown in LB broth (ForMedium) containing kanamycin $\left(30 \mu \mathrm{g} \mathrm{mL}^{-1}\right)$ and incubated at $37{ }^{\circ} \mathrm{C}$ with $190 \mathrm{rpm}$ shaking, until an $\mathrm{OD}_{600}$ of 0.3 was reached. Recombinant protein production was induced by the addition of $20 \%(\mathrm{w} / \mathrm{v})$ rhamnose, $10 \%(\mathrm{w} / \mathrm{v})$ arabinose, and $5 \mathrm{mM}$ biotin solutions, to obtain final concentrations of $0.2 \%(\mathrm{w} / \mathrm{v})$, $0.01 \%(\mathrm{w} / \mathrm{v})$, and $50 \mu \mathrm{M}$, respectively. After $24 \mathrm{~h}$ the cells were harvested by centrifugation at $6000 \mathrm{~g}, 4{ }^{\circ} \mathrm{C}$, for $10 \mathrm{~min}$, and the pellet was resuspended in Buffer A supplemented with DNase $\left(0.1 \mathrm{mg} \mathrm{mL}^{-1}\right)$, lysozyme $\left(0.1 \mathrm{mg} \mathrm{mL}^{-1}\right)$, and an EDTA free protease inhibitor tablet. The harvested cells were then lysed and purified as described by Karuppiah et al. (2017).

For the production of anti-bLinS $\mathrm{V}_{\mathrm{HH}}$, the appropriate chemically competent cells were transformed with the $\mathrm{pBbE8k}$ $\mathrm{His}_{6}-\mathrm{V}_{\mathrm{HH}} \mathrm{CyDisCo}$ variants. Single colonies were used to 
inoculate $2 \mathrm{~mL}$ of $\mathrm{LB}$ overnight cultures supplemented with kanamycin $\left(50 \mu \mathrm{g} \mathrm{mL}^{-1}\right)$. These initial cultures were incubated overnight at $30{ }^{\circ} \mathrm{C}$ with shaking at $200 \mathrm{rpm}$ and used to inoculate $\mathrm{TB}$ supplemented with kanamycin $\left(50 \mu \mathrm{g} \mathrm{mL}^{-1}\right)$ as a 1:100 dilution. The cultures were grown at $30{ }^{\circ} \mathrm{C}$ with shaking $(200 \mathrm{rpm})$ until an $\mathrm{OD}_{600}$ of 0.6 was reached. The cultures were then induced by the addition of arabinose to a final concentration of $50 \mathrm{mM}$, and the temperature was subsequently altered to $26.6{ }^{\circ} \mathrm{C}$. The induced cultures were then incubated for a further $24 \mathrm{~h}$, before centrifugation and removal of the supernatant. Cell pellets were then flash frozen in liquid nitrogen and stored at $-80{ }^{\circ} \mathrm{C}$ or immediately taken forward for cell lysis. Lysis buffer $(25 \mathrm{mM}$ Tris- $\mathrm{HCl} \mathrm{pH} 7.4$, $150 \mathrm{mM} \mathrm{NaCl}, 5 \%$ (v/v) glycerol, $0.1 \mathrm{mg} \mathrm{mL}^{-1} \mathrm{DNaseI}$, and $0.1 \mathrm{mg} \mathrm{mL}^{-1}$ lysozyme) was added to the frozen pellet at 1 / 10th of the initial culture volume, and the cell pellet was resuspended by vortexing. Three additional freeze-thaw cycles were performed to ensure thorough lysis of cells. The lysate was then clarified by centrifugation at $13000 \mathrm{~g}$ for $10 \mathrm{~min}$, and the supernatant was removed and retained for further analysis/ purification.

Protein purity was monitored by SDS-PAGE. Protein concentration was determined using both the Bradford protein assay (Bio-Rad) and extinction coefficient methodology.

Construction of anti-bLinS Phage Display Library. A single male llama (Llama glama) was provided for use by UCB as part of their collaboration with the University of Reading. All llama handling steps were performed by a trained expert according to protocols compliant with the Animal (Scientific Procedures) Act 1986. The llama was given five subcutaneous injections of purified Streptomyces clavuligerus linalool synthase (bLinS) at monthly intervals, with bleeds $(\sim 500 \mathrm{~mL})$ taken after the third (Bleed 1) and fifth (Bleed 2) injections. bLinS (purified as described above) was prepared as five $500 \mu \mathrm{L}$ aliquots $\left(2 \mathrm{mg} \mathrm{mL}^{-1}\right)$ which were mixed 1:1 with GERBU adjuvant prior to immunization.

During the library construction process Bleed 1 and Bleed 2 reactions were performed independently, such that two libraries were obtained. B-cell mRNA was isolated from PBMC (Bleed 1:5 $510^{8}$ cells, Bleed 2:3 $\times 10^{8}$ cells) using a Qiagen TissueRuptor and RNeasy plus midi kit. mRNA isolated from each library was taken forward for RT-PCR using SuperScript IV reverse transcriptase (Invitrogen) with both oligo-dT and random hexamer (dN6) primers. Primary PCR amplification of the oligo-dT and dN6 generated cDNA between the signal peptide and $\mathrm{C}_{\mathrm{H}} 2$ domain of IgG/HCAb coding sequences was achieved using KOD Polymerase (Merck Millipore) with primers CaLL01 and CaLL02 (Supplementary Table 5). The oligo dT and dN6 primary PCR products were pooled, and the $\mathrm{V}_{\mathrm{HH}}$ population (600 bp) was purified. The purified $\mathrm{V}_{\mathrm{HH}}$ amplicons were used as template for a secondary PCR using Herculase II polymerase (Agilent) with primers $\mathrm{V}_{\mathrm{HH}} \mathrm{Sh} \mathrm{Fw}$ and CldLib2Rv-01 or CldLib2Rv-12, for Bleeds 1 and 2, respectively (Supplementary Table 5). Secondary PCR products were purified and digested, alongside the phagemid vector (provided by UCB Pharma), using NotI and SfiI endonucleases. Ligation of the digested phagemid vector $(4 \mu \mathrm{g})$ and $\mathrm{V}_{\mathrm{HH}}$ insert $(1 \mu \mathrm{g})$ was performed using T4 DNA Ligase (New England Biolabs).

Electrocompetent TG-1 cells (Lucigen) were transformed with the purified ligation product. Following the recovery of cells, the transformation mixtures were pooled, and serial dilutions $\left(10^{-1}, 10^{-2}, 10^{-3}, 10^{-4}, 10^{-5}\right)$ of the recovered transformants were spread onto 2X YT Agar, 1\% glucose, 100 $\mu \mathrm{g} \mathrm{mL}^{-1}$ carbenicillin plates, and incubated overnight at $37{ }^{\circ} \mathrm{C}$ such that the library size could be estimated. The remaining transformation mixture was pelleted and resuspended in $1 \mathrm{~mL}$ of LB medium. The cell slurry was then spread across three $\mathrm{XL}$ Bio dishes (2X YT Agar, 1\% glucose, $100 \mu \mathrm{g} \mathrm{mL}^{-1}$ carbenicillin) per library and incubated overnight at $30{ }^{\circ} \mathrm{C}$. The following day each XL Bio dish was washed with $2 \mathrm{X}$ YT, $1 \%$ glucose, $100 \mu \mathrm{g} \mathrm{mL}^{-1}$ carbenicillin and the cell slurry collected. The slurry was used to inoculate a fresh culture of

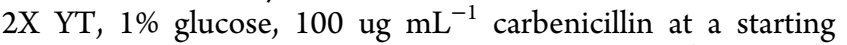
$\mathrm{OD}_{600}$ of 0.1 and grown at $37{ }^{\circ} \mathrm{C}$, with shaking $(200 \mathrm{rpm})$, until an $\mathrm{OD}_{600}$ of 0.5 was reached. M13KO7 helper phage, at a multiplicity of infection (MOI) of 20, was used for library rescue as per established protocols. ${ }^{50,51}$ Phage particles were recovered from the culture supernatant by precipitation using $20 \%$ PEG 8000, $2.5 \mathrm{M} \mathrm{NaCl}$. The purified phage were resuspended in PBS, $20 \%$ glycerol. Library size was calculated by phage infective titering.

Enrichment of anti-bLinS Phage Display Library. For each panning condition, sufficient phage particles were added so as to cover the estimated diversity of the libraries 20,000 fold. The phage libraries were blocked in an equal volume of $3 \%$ BSA, 3\% milk powder in PBS for $1 \mathrm{~h}$ at room temperature with rolling. Simultaneously, NeutrAvidin coated Nunc Maxisorp 96 well plates (Invitrogen) or Dynabeads M-280 Streptavidin (Invitrogen) were blocked with 1\% BSA, PBS for $1 \mathrm{~h}$ with shaking/rolling. Biotinylated bLinS $\left(2 \mu \mathrm{g} \mathrm{mL}{ }^{-1}\right)$ in $1 \%$ BSA, PBS was then added to the blocked libraries for each panning condition and incubated for $1 \mathrm{~h}$. The biotinylated bLinS-phage mixture (and a phage only control) was subsequently added to the coated microtiter plate/Dynabead slurry and incubated for $1 \mathrm{~h}$ with gentle agitation. The coated microtiter plate/Dynabead slurry (Dynabeads were pelleted using a DynaMag-2 magnet) was then washed using PBS $0.1 \%$ Tween-20 $(5 \times 300 \mu \mathrm{L}$ for microtiter plate and $10 \times 2 \mathrm{~mL}$ for Dynabeads), followed by an additional two washes $(300 \mu \mathrm{L}$ and $2 \mathrm{~mL}$ for microtiter plate and Dynabeads respectively) using PBS. The bound phage particles were eluted by the addition of trypsin $\left(1 \mu \mathrm{g} \mathrm{mL}^{-1}\right.$ in PBS, $\left.1 \mathrm{mM} \mathrm{CaCl}_{2}\right)$ which was added to the dried microtiter plate/Dynabeads and left to incubate at room temperature with shaking $(600 \mathrm{rpm})$ for 30 min. The trypsin treated supernatant containing the eluted phage particles were then allowed to infect E. coli TG1 cells grown in 2X YT Broth (ForMedium) at an $\mathrm{OD}_{600}$ of $0.2-0.4$. The infected TG1 cells were incubated (static) at $37{ }^{\circ} \mathrm{C}$ for 30 $\min$. In order to estimate the output phage diversity, a serial dilution $\left(10^{-1}\right.$ to $\left.10^{-5}\right)$ of the infected TG1 cells in $2 \mathrm{X}$ YT was performed, with the dilutions plated onto small LB agar plates supplemented with $1 \%$ glucose and $100 \mu \mathrm{g} \mathrm{mL}^{-1}$ carbenicillin. The output titers were subsequently estimated by counting the colonies present at each dilution after $16 \mathrm{~h}$ of incubation at 37 ${ }^{\circ} \mathrm{C}$.

Small scale monoclonal phage rescue of individual colonies from the output phage titer plates obtained following library enrichment was performed using M13KO7 helper phage (New England Biolabs). Binding of enriched Bleed 1 and Bleed 2 monoclonal $\mathrm{V}_{\mathrm{HH}}$ to biotinylated bLinS was confirmed by ELISA. Following the identification of biotinylated bLinS binders, fresh 96 deep-well culture blocks containing LB medium supplemented with $100 \mu \mathrm{g} \mathrm{mL}^{-1}$ carbenicillin and $2 \%$ glucose were inoculated using the initial monoclonal rescue blocks as a 1:100 inoculum. Following overnight growth the 
phagemid vector was isolated using a 96-well plate plasmid DNA Miniprep kit (Bio Basic) and a 96-well plate vacuum manifold (Phenomenex). The phagemid vectors were sequenced using LMB3 and FDSEQ primers (Supplementary Table 5), and the translated sequences were aligned using the CLUSTAL Omega ${ }^{52}$ sequence alignment tool. Sequence logos were created using the WebLogo server version 2.8.2.2. ${ }^{53}$

Design of Experiments and PLS Regression Analysis. Design of Experiments, statistical analysis and partial leastsquares (PLS) regression was performed using JMP Pro 12 (SAS Institute). The Custom Design tool was used to create an I-optimal design that investigated all main effects (single effects of the variables themselves), two-factor interactions (interactions between two variables), and power terms for the continuous variables (an interaction of a continuous variable against itself in order to test for curvature). The experimental design consisted of a mixture of categorical and continuous input variables (experimental factors). The categorical variables used in the design were $\mathrm{N}$-terminal tag (None, $\mathrm{His}_{6}, \mathrm{His}_{6}-\mathrm{TEV}$, and $\mathrm{His}_{6} \mathrm{~S}$-tag), CyDisCo (No, Yes), and strain (NEB10 $\beta$, NiCo21 (DE3), T7 Express, and SHuffle T7 Express). The continuous variables were arabinose concentration $(0.1-50 \mathrm{mM})$ and postinduction temperature $\left(16^{\circ} \mathrm{C}-\right.$ $\left.30{ }^{\circ} \mathrm{C}\right)$. The cytoplasmic production of functional anti-LinS $\mathrm{V}_{\mathrm{HH}}$ was determined by ELISA; as such the absorbance at 630 $\mathrm{nm}$ minus the absorbance at $490 \mathrm{~nm}\left(\Delta A_{630-490}\right)$ was used as the response variable. The resultant design consisted of 42 experiments (Supplementary Table 2) which were performed in parallel in biological triplicate $(n=3)$. N-terminal tag and CyDisCo sequences can be found in Supplementary Tables 1 and 6 , respectively.

Modeling of the experimental data was performed by PLSR. Model validation was performed using leave-one-out cross validation (LOOCV) and the SIMPLS (statistically inspired modification of the PLS method) ${ }^{32}$ algorithm. The root mean predicted residual error sum of squares (RM-PRESS) statistic was used to determine the optimal number of factors (latent variables) within the model. The variable importance in projection (VIP) and coefficient values for each variable within the model were then used to identify those that had the greatest effect. ${ }^{54}$ Variables with a VIP $>0.8$ were taken forward to fit a second iteration of the PLSR model which was validated using the methods described above.

Enzyme-Linked Immunosorbent Assays (ELISA). Wash steps utilized PBS $0.1 \%(\mathrm{v} / \mathrm{v})$ Tween-20, while the incubation of the plates between steps was performed at room temperature for $1 \mathrm{~h}$ with shaking. Nunc Maxisorp 96-well plates were coated with $50 \mu \mathrm{L}$ of NeutrAvidin or Streptavidin (1 $\mu \mathrm{g} \mathrm{mL} L^{-1} \mathrm{PBS}$ ) and incubated at $4{ }^{\circ} \mathrm{C}$ overnight. The following day all plates were washed and blocked for $1 \mathrm{~h}$ at room temperature using $1 \% \mathrm{BSA}$ in PBS. The blocked plates were again washed, and $50 \mu \mathrm{L}$ of biotinylated bLinS $(1 \mu \mathrm{g}$ $\mathrm{mL}^{-1}$ in PBS, $5 \%(\mathrm{v} / \mathrm{v})$ glycerol and $5 \mathrm{mM} \mathrm{MgCl}_{2}$ ) was added prior to an additional incubation period. After a further washing step, cell lysate was added in triplicate $(n=3)$. For titration ELISA, lysate was diluted in Buffer A prior to addition to the blocked plates. For monoclonal phage rescue ELISAs, supernatant containing monoclonal phage displaying antibLinS $\mathrm{V}_{\mathrm{HH}}$ was blocked in an equal volume of PBS $2 \%$ BSA $2 \%$ milk powder, prior to addition onto the biotinylated bLinS coated plates. Following addition of anti-bLinS $\mathrm{V}_{\mathrm{HH}}$, the plates were incubated for $1 \mathrm{~h}$ before being washed. Bound $\mathrm{V}_{\mathrm{HH}}$ was revealed using MonoRab anticamelid $\mathrm{V}_{\mathrm{HH}}$ HRP-conjugated monoclonal antibody (GenScript) at a 1:5000 dilution in PBS. Following the addition of 1 Step Ultra TMB ELISA Substrate (ThermoFisher) the absorbance at 630 and $490 \mathrm{~nm}$ was measured using a BioTek Synergy HT microplate reader. Antibody binding to the immobilized antigen was calculated by subtracting the absorbance at $490 \mathrm{~nm}$ from the absorbance at $630 \mathrm{~nm}\left(\Delta A_{630-490}\right)$.

Production of Monoterpenoids In Vivo. For the production of monoterpenoids in vivo NEB $10 \beta$, NEB $5 \alpha$, BL21 (DE3), T7 Express, and SHuffle T7 Express were transformed with pJBEI-6410 bLinS. Freshly transformed colonies were then used to inoculate LB (ForMedium) supplemented with $100 \mathrm{mg} \mathrm{mL} \mathrm{mL}^{-1}$ carbenicillin, which were grown overnight at $30{ }^{\circ} \mathrm{C}$. Overnight cultures were used to inoculate (1:100 dilution) TB (ForMedium) supplemented with $1 \%(\mathrm{v} / \mathrm{v})$ glucose or glycerol and $100 \mathrm{mg} \mathrm{mL}^{-1}$ carbenicillin in glass screw capped vials $(2 \mathrm{~mL}$ reaction volume) which were incubated at $30{ }^{\circ} \mathrm{C}$ with shaking at 200 $\mathrm{rpm}$. Once an optical density $\left(\mathrm{OD}_{600}\right)$ of 0.6 was reached linalool production was induced with $100 \mu \mathrm{M}$ IPTG. Following induction, a $20 \%(\mathrm{v} / \mathrm{v}) n$-nonane overlay was also added so as to capture the volatile monoterpenoids produced. The cultures were incubated for a further $72 \mathrm{~h}$, before the organic layer was collected and dried using anhydrous $\mathrm{MgSO}_{4}$. The dried organic phase was then mixed at a $1: 1$ ratio withethyl acetate containing $0.01 \%$ sec-butyl benzene as an internal standard. The samples were then analyzed by GCMS.

When assessing the modulatory effect of $\mathrm{V}_{\mathrm{HH}}$ in vivo, competent cells were cotransformed with pJBEI-6410 bLinS and pBbE8k His ${ }_{6}-\mathrm{V}_{\mathrm{HH}}$ CyDisCo. Single colonies were used to establish overnight cultures, which were in turn used to establish production cultures as described above, with both the overnight and production media additionally supplemented with $50 \mathrm{mg} \mathrm{mL}^{-1}$ kanamycin. Once an $\mathrm{OD}_{600}$ of $\sim 0.6$ was reached monoterpenoid production was induced by the addition of $100 \mu \mathrm{M}$ IPTG while $\mathrm{V}_{\mathrm{HH}}$ production was induced using $2 \mathrm{mM}$ arabinose. The remaining steps were performed as described above.

GC-MS analysis. Samples were injected onto an Agilent Technologies 7890AB GC coupled to an Agilent 5975A MSD. The products were separated using a DB-WAX column $(30 \mathrm{~m}$; $0.32 \mathrm{~mm} ; 0.25 \mu \mathrm{m}$ film thickness, JW Scientific). The injector temperature was $220{ }^{\circ} \mathrm{C}$ with a split ratio of 50:1 $(1 \mu \mathrm{L}$ injection). The carrier gas was helium with a flow rate of $3 \mathrm{~mL}$ $\mathrm{min}^{-1}$ and a pressure of $8.3 \mathrm{psi}$. The oven program began at 40 ${ }^{\circ} \mathrm{C}$ with a hold for $2 \mathrm{~min}$ followed by an increase of temperature to $70{ }^{\circ} \mathrm{C}$ at a rate of $6{ }^{\circ} \mathrm{C} / \mathrm{min}$; after this point the temperature was increased to $210{ }^{\circ} \mathrm{C}$ at a rate of $50{ }^{\circ} \mathrm{C} / \mathrm{min}$ with a final hold at $210{ }^{\circ} \mathrm{C}$ for $2 \mathrm{~min}$. The ion source temperature of the mass spectrometer was set to $210^{\circ} \mathrm{C}$, and spectra were recorded form $\mathrm{m} / z 50$ to $\mathrm{m} / z 250$. The mass spectra fragmentation patterns that were obtained were entered into the NIST mass spectral library for identification, product identity was also confirmed by the use of commercially bought standards. Quantification of the products was calculated using the ratios between the internal standard and the standard prepared at a known concentration.

\section{ASSOCIATED CONTENT}

\section{SI Supporting Information}

The Supporting Information is available free of charge at https://pubs.acs.org/doi/10.1021/acssynbio.0c00375. 
Additional information relating to $\mathrm{V}_{\mathrm{HH}}$ sequences obtained from immune libraries, DoE and PLSR model building, screening of optimized production conditions, and primers used in this work (PDF)

\section{AUTHOR INFORMATION}

\section{Corresponding Author}

Nigel S. Scrutton - Manchester Institute of Biotechnology, Department of Chemistry, School of Natural Sciences, The University of Manchester, Manchester M13 9PL, United Kingdom; (1) orcid.org/0000-0002-4182-3500; Email: nigel.scrutton@manchester.ac.uk

\section{Authors \\ Jonathan Wilkes - Manchester Institute of Biotechnology, Department of Chemistry, School of Natural Sciences, The University of Manchester, Manchester M13 9PL, United Kingdom \\ Anthony Scott-Tucker - UCB Pharma Ltd., Slough SL1 3WE, United Kingdom \\ Mike Wright - UCB Pharma Ltd., Slough SL1 3WE, United Kingdom \\ Tom Crabbe - UCB Pharma Ltd., Slough SL1 3WE, United Kingdom}

Complete contact information is available at:

https://pubs.acs.org/10.1021/acssynbio.0c00375

\section{Author Contributions}

J.W., A.S.T., M.W., T.C., and N.S.S. wrote and reviewed the manuscript text. Figures were prepared by J.W. Phage display libraries were constructed by J.W. and A.S.T. using methods developed by A.S.T. and M.W. All remaining experimental work was proposed, designed, and performed by J.W.

\section{Notes}

The authors declare no competing financial interest.

\section{ACKNOWLEDGMENTS}

This research was funded by a BBSRC CASE award with UCB Pharma (BB/N503976/1). The plasmid pJBEI-6410 was a gift from Taek Soon Lee (Addgene plasmid \#47049).

\section{REFERENCES}

(1) Böldicke, T. (2017) Single Domain Antibodies for the Knockdown of Cytosolic and Nuclear Proteins. Protein Sci. 26 (5), 925-945.

(2) Tang, J. C. Y., Szikra, T., Kozorovitskiy, Y., Teixiera, M., Sabatini, B. L., Roska, B., and Cepko, C. L. (2013) A Nanobody-Based System Using Fluorescent Proteins as Scaffolds for Cell-Specific Gene Manipulation. Cell 154 (4), 928-939.

(3) Prole, D. L., and Taylor, C. W. (2019) A Genetically Encoded Toolkit of Functionalized Nanobodies against Fluorescent Proteins for Visualizing and Manipulating Intracellular Signalling. BMC Biol. 17 (1), 41.

(4) Harmansa, S., and Affolter, M. (2018) Protein Binders and Their Applications in Developmental Biology. Development 145 (2), No. dev148874.

(5) Gąciarz, A., Veijola, J., Uchida, Y., Saaranen, M. J., Wang, C., Hörkkö, S., and Ruddock, L. W. (2016) Systematic Screening of Soluble Expression of Antibody Fragments in the Cytoplasm of E. coli. Microb. Cell Fact. 15 (1), 22.

(6) Plückthun, A. (2015) Designed Ankyrin Repeat Proteins (DARPins): Binding Proteins for Research, Diagnostics, and Therapy. Annu. Rev. Pharmacol. Toxicol. 55 (1), 489-511.
(7) Koide, A., Wojcik, J., Gilbreth, R. N., Hoey, R. J., and Koide, S. (2012) Teaching an Old Scaffold New Tricks: Monobodies Constructed Using Alternative Surfaces of the FN3 Scaffold. J. Mol. Biol. 415 (2), 393-405.

(8) Ståhl, S., Gräslund, T., Eriksson Karlström, A., Frejd, F. Y., Nygren, P.-A., and Löfblom, J. (2017) Affibody Molecules in Biotechnological and Medical Applications. Trends Biotechnol. 35 (8), 691-712.

(9) Gebauer, M., and Skerra, A. (2012) Anticalins: Small Engineered Binding Proteins Based on the Lipocalin Scaffold. Methods Enzymol. 503, 157-188.

(10) Tiede, C., Bedford, R., Heseltine, S. J., Smith, G., Wijetunga, I., Ross, R., AlQallaf, D., Roberts, A. P., Balls, A., Curd, A., Hughes, R. E., Martin, H., Needham, S. R., Zanetti-Domingues, L. C., Sadigh, Y., Peacock, T. P., Tang, A. A., Gibson, N., Kyle, H., Platt, G. W., Ingram, N., Taylor, T., Coletta, L. P., Manfield, I., Knowles, M., Bell, S., Esteves, F., Maqbool, A., Prasad, R. K., Drinkhill, M., Bon, R. S., Patel, V., Goodchild, S. A., Martin-Fernandez, M., Owens, R. J., Nettleship, J. E., Webb, M. E., Harrison, M., Lippiat, J. D., Ponnambalam, S., Peckham, M., Smith, A., Ferrigno, P. K., Johnson, M., McPherson, M. J., and Tomlinson, D. C. (2017) Affimer Proteins Are Versatile and Renewable Affinity Reagents. eLife 6, No. 24903, DOI: 10.7554/ eLife.24903.

(11) Helma, J., Cardoso, M. C., Muyldermans, S., and Leonhardt, H. (2015) Nanobodies and Recombinant Binders in Cell Biology. J. Cell Biol. 209 (5), 633-644.

(12) Henry, K. A., and MacKenzie, C. R. (2018) Editorial: SingleDomain Antibodies-Biology, Engineering and Emerging Applications. Front. Immunol. 9, 41.

(13) Lobstein, J., Emrich, C. A., Jeans, C., Faulkner, M., Riggs, P., and Berkmen, M. (2012) SHuffle, a Novel Escherichia coli Protein Expression Strain Capable of Correctly Folding Disulfide Bonded Proteins in Its Cytoplasm. Microb. Cell Fact. 11 (1), 753.

(14) Gaciarz, A., Khatri, N. K., Velez-Suberbie, M. L., Saaranen, M. J., Uchida, Y., Keshavarz-Moore, E., and Ruddock, L. W. (2017) Efficient Soluble Expression of Disulfide Bonded Proteins in the Cytoplasm of Escherichia coli in Fed-Batch Fermentations on Chemically Defined Minimal Media. Microb. Cell Fact. 16 (1), 108.

(15) Nguyen, V., Hatahet, F., Salo, K. E., Enlund, E., Zhang, C., and Ruddock, L. W. (2011) Pre-Expression of a Sulfhydryl Oxidase Significantly Increases the Yields of Eukaryotic Disulfide Bond Containing Proteins Expressed in the Cytoplasm of E. coli. Microb. Cell Fact. 10 (1), 1.

(16) Hatahet, F., Nguyen, V. D., Salo, K. E. H., and Ruddock, L. W. (2010) Disruption of Reducing Pathways Is Not Essential for Efficient Disulfide Bond Formation in the Cytoplasm of E. coli. Microb. Cell Fact. 9, 67.

(17) Miller, L. H., and Su, X. (2011) Artemisinin: Discovery from the Chinese Herbal Garden. Cell 146 (6), 855-858.

(18) Leferink, N. G. H., Dunstan, M. S., Hollywood, K. A., Swainston, N., Currin, A., Jervis, A. J., Takano, E., and Scrutton, N. S. (2019) An Automated Pipeline for the Screening of Diverse Monoterpene Synthase Libraries. Sci. Rep. 9 (1), 11936.

(19) Duetz, W. A., Bouwmeester, H., Beilen, J. B., and Witholt, B. (2003) Biotransformation of Limonene by Bacteria, Fungi, Yeasts, and Plants. Appl. Microbiol. Biotechnol. 61 (4), 269-277.

(20) Zebec, Z., Wilkes, J., Jervis, A. J., Scrutton, N. S., Takano, E., and Breitling, R. (2016) Towards Synthesis of Monoterpenes and Derivatives Using Synthetic Biology. Curr. Opin. Chem. Biol. 34, 3743.

(21) Leferink, N. G. H., Jervis, A. J., Zebec, Z., Toogood, H. S., Hay, S., Takano, E., and Scrutton, N. S. (2016) A 'Plug and Play' Platform for the Production of Diverse Monoterpene Hydrocarbon Scaffolds in Escherichia coli. ChemistrySelect 1 (9), 1893-1896.

(22) George, K. W., Alonso-Gutierrez, J., Keasling, J. D., and Lee, T. S. (2015) Isoprenoid Drugs, Biofuels, and Chemicals-Artemisinin, Farnesene, and Beyond. Adv. Biochem. Eng./Biotechnol. 123, 355-389.

(23) Govindarajan, S., Mannervik, B., Silverman, J. A., Wright, K., Regitsky, D., Hegazy, U., Purcell, T. J., Welch, M., Minshull, J., and 
Gustafsson, C. (2015) Mapping of Amino Acid Substitutions Conferring Herbicide Resistance in Wheat Glutathione Transferase. ACS Synth. Biol. 4 (3), 221-227.

(24) Brown, S. R., Staff, M., Lee, R., Love, J., Parker, D. A., Aves, S. J., and Howard, T. P. (2018) Design of Experiments Methodology to Build a Multifactorial Statistical Model Describing the Metabolic Interactions of Alcohol Dehydrogenase Isozymes in the Ethanol Biosynthetic Pathway of the Yeast Saccharomyces cerevisiae. ACS Synth. Biol. 7 (7), 1676-1684.

(25) Xu, P., Rizzoni, E. A., Sul, S.-Y., and Stephanopoulos, G. (2017) Improving Metabolic Pathway Efficiency by Statistical Model-Based Multivariate Regulatory Metabolic Engineering. ACS Synth. Biol. 6 (1), 148-158.

(26) Kent, R., and Dixon, N. (2019) Systematic Evaluation of Genetic and Environmental Factors Affecting Performance of Translational Riboswitches. ACS Synth. Biol. 8 (4), 884-901.

(27) Esposito, D., and Chatterjee, D. K. (2006) Enhancement of Soluble Protein Expression through the Use of Fusion Tags. Curr. Opin. Biotechnol. 17 (4), 353-358.

(28) Eliseev, I. E., Yudenko, A. N., Vysochinskaya, V. V., Svirina, A. A., Evstratyeva, A. V., Drozhzhachih, M. S., Krendeleva, E. A., Vladimirova, A. K., Nemankin, T. A., Ekimova, V. M., Ulitin, A. B., Lomovskaya, M. I., Yakovlev, P. A., Bukatin, A. S., Knyazev, N. A., Moiseenko, F. V., and Chakchir, O. B. (2018) Open Peer Review Crystal Structures of a Llama VHH Antibody BCD090-M2 Targeting Human ErbB3 Receptor [Version 2; Peer Review: 2 Approved]. F1000Research 7, 57.

(29) Hinz, A., Lutje Hulsik, D., Forsman, A., Koh, W. W.-L., Belrhali, H., Gorlani, A., de Haard, H., Weiss, R. A., Verrips, T., and Weissenhorn, W. (2010) Crystal Structure of the Neutralizing Llama VHH D7 and Its Mode of HIV-1 Gp120 Interaction. PLoS One 5 (5), No. e10482.

(30) Larentis, A. L., Nicolau, J. F. M. Q., Esteves, G. d. S., Vareschini, D. T., de Almeida, F. V. R., dos Reis, M. G., Galler, R., and Medeiros, M. A. (2014) Evaluation of Pre-Induction Temperature, Cell Growth at Induction and IPTG Concentration on the Expression of a Leptospiral Protein in E. coli Using Shaking Flasks and Microbioreactor. BMC Res. Notes 7 (1), 671.

(31) Mühlmann, M., Forsten, E., Noack, S., and Büchs, J. (2017) Optimizing Recombinant Protein Expression via Automated Induction Profiling in Microtiter Plates at Different Temperatures. Microb. Cell Fact. 16 (1), 220.

(32) de Jong, S. (1993) SIMPLS: An Alternative Approach to Partial Least Squares Regression. Chemom. Intell. Lab. Syst. 18 (3), 251-263.

(33) Willrodt, C., David, C., Cornelissen, S., Bühler, B., Julsing, M. K., and Schmid, A. (2014) Engineering the Productivity of Recombinant Escherichia coli for Limonene Formation from Glycerol in Minimal Media. Biotechnol. J. 9 (8), 1000-1012.

(34) Liu, W., Zhang, R., Tian, N., Xu, X., Cao, Y., Xian, M., and Liu, H. (2015) Utilization of Alkaline Phosphatase PhoA in the Bioproduction of Geraniol by Metabolically Engineered Escherichia coli. Bioengineered 6 (5), 288-293.

(35) Anthony, J. R., Anthony, L. C., Nowroozi, F., Kwon, G., Newman, J. D., and Keasling, J. D. (2009) Optimization of the Mevalonate-Based Isoprenoid Biosynthetic Pathway in Escherichia coli for Production of the Anti-Malarial Drug Precursor Amorpha-4,11Diene. Metab. Eng. 11 (1), 13-19.

(36) Martin, V. J. J., Pitera, D. J., Withers, S. T., Newman, J. D., and Keasling, J. D. (2003) Engineering a Mevalonate Pathway in Escherichia coli for Production of Terpenoids. Nat. Biotechnol. 21 (7), 796-802.

(37) Pitera, D. J., Paddon, C. J., Newman, J. D., and Keasling, J. D. (2007) Balancing a Heterologous Mevalonate Pathway for Improved Isoprenoid Production in Escherichia coli. Metab. Eng. 9 (2), 193-207.

(38) Karuppiah, V., Ranaghan, K., Leferink, N. G. H., Johannissen, L. O., Shanmugam, M., Ni Cheallaigh, A., Bennett, N., Kearsey, L., Takano, E., Gardiner, J., Van der Kamp, M., Hay, S., Mulholland, A. J., Leys, D., and Scrutton, N. S. (2017) Structural Basis of Catalysis in the Bacterial Monoterpene Synthases Linalool Synthase and 1,8Cineole Synthase. ACS Catal. 7, 6268.

(39) Fujisaki, S., Nishino, T., and Katsuki, H. (1986) Isoprenoid Synthesis in Escherichia coli. Separation and Partial Purification of Four Enzymes Involved in the Synthesis1. J. Biochem. 99 (5), 13271337.

(40) Ajikumar, P. K., Xiao, W.-H., Tyo, K. E. J., Wang, Y., Simeon, F., Leonard, E., Mucha, O., Phon, T. H., Pfeifer, B., and Stephanopoulos, G. (2010) Isoprenoid Pathway Optimization for Taxol Precursor Overproduction in Escherichia coli. Science 330 (6000), 70-74.

(41) Alonso-Gutierrez, J., Kim, E.-M., Batth, T. S., Cho, N., Hu, Q., Chan, L. J. G., Petzold, C. J., Hillson, N. J., Adams, P. D., Keasling, J. D., Garcia-Martin, H., and Soon Lee, T. (2015) Principal Component Analysis of Proteomics (PCAP) as a Tool to Direct Metabolic Engineering. Metab. Eng. 28, 123-133.

(42) Zhou, J., Wang, C., Yang, L., Choi, E.-S., and Kim, S.-W. (2015) Geranyl Diphosphate Synthase: An Important Regulation Point in Balancing a Recombinant Monoterpene Pathway in Escherichia coli. Enzyme Microb. Technol. 68, 50-55.

(43) Sarria, S., Wong, B., García Martín, H., Keasling, J. D., and Peralta-Yahya, P. (2014) Microbial Synthesis of Pinene. ACS Synth. Biol. 3 (7), 466-475.

(44) Marschall, A. L., Dübel, S., and Böldicke, T. (2015) Specific in Vivo Knockdown of Protein Function by Intrabodies. MAbs 7 (6), $1010-1035$

(45) Alonso-Gutierrez, J., Chan, R., Batth, T. S., Adams, P. D., Keasling, J. D., Petzold, C. J., and Lee, T. S. (2013) Metabolic Engineering of Escherichia coli for Limonene and Perillyl Alcohol Production. Metab. Eng. 19, 33-41.

(46) Espah Borujeni, A., Channarasappa, A. S., and Salis, H. M. (2014) Translation Rate Is Controlled by Coupled Trade-Offs between Site Accessibility, Selective RNA Unfolding and Sliding at Upstream Standby Sites. Nucleic Acids Res. 42 (4), 2646-2659.

(47) Salis, H. M., Mirsky, E. A., and Voigt, C. A. (2009) Automated Design of Synthetic Ribosome Binding Sites to Control Protein Expression. Nat. Biotechnol. 27 (10), 946-950.

(48) Giacalone, M. J., Gentile, A. M., Lovitt, B. T., Berkley, N. L., Gunderson, C. W., and Surber, M. W. (2006) Toxic Protein Expression in Escherichia coli Using a Rhamnose-Based Tightly Regulated and Tunable Promoter System. BioTechniques 40 (3), 355364.

(49) Lee, T. S., Krupa, R. A., Zhang, F., Hajimorad, M., Holtz, W. J., Prasad, N., Lee, S. K., and Keasling, J. D. (2011) BglBrick Vectors and Datasheets: A Synthetic Biology Platform for Gene Expression. J. Biol. Eng. 5 (1), 12.

(50) Zhu, Z., and Dimitrov, D. S. (2009) Construction of a Large Naive Human Phage-Displayed Fab Library Through One-Step Cloning. Methods Mol. Biol. 525, 129-142.

(51) de Haard, H. J. W. (2002) Construction of Large Nä̈ve Fab Libraries. Antibody Phage Display, 087-100, DOI: 10.1385/1-59259240-6:087.

(52) Madeira, F., Park, Y. M., Lee, J., Buso, N., Gur, T., Madhusoodanan, N., Basutkar, P., Tivey, A. R. N., Potter, S. C., Finn, R. D., and Lopez, R. (2019) The EMBL-EBI Search and Sequence Analysis Tools APIs in 2019. Nucleic Acids Res. 47, W636.

(53) Crooks, G. E. (2004) WebLogo: A Sequence Logo Generator. Genome Res. 14 (6), 1188-1190.

(54) Wold, S., Sjöström, M., and Eriksson, L. (2001) PLSRegression: A Basic Tool of Chemometrics. Chemom. Intell. Lab. Syst. 58 (2), 109-130. 